\title{
BMJ Open In-hospital cardiac rehabilitation and clinical outcomes in patients with acute myocardial infarction after percutaneous coronary intervention: a retrospective cohort study
}

\author{
Natsuko Kanazawa (D) , ${ }^{1,2}$ Hiroaki lijima, ${ }^{3}$ Kiyohide Fushimi ${ }^{1,2}$
}

To cite: Kanazawa N, lijima H, Fushimi K. In-hospital cardiac rehabilitation and clinical outcomes in patients with acute myocardial infarction after percutaneous coronary intervention: a retrospective cohort study. BMJ Open 2020;10:e039096. doi:10.1136/ bmjopen-2020-039096

- Prepublication history for this paper is available online. To view these files, please visit the journal online (http://dx.doi. org/10.1136/bmjopen-2020039096).

Received 03 April 2020 Revised 14 August 2020 Accepted 19 August 2020

Check for updates

(C) Author(s) (or their employer(s)) 2020. Re-use permitted under CC BY-NC. No commercial re-use. See rights and permissions. Published by BMJ.

${ }^{1}$ Department of Health Policy and Informatics, Graduate

School of Medical and Dental

Sciences, Tokyo Medical and Dental University, Bunkyo-ku, Japan

${ }^{2}$ Department of Clinical Data Management and Research, Clinical Research Center, National Hospital Organization, Meguro, Japan

${ }^{3}$ Biostatistics Division, Clinical Research and Medical Innovation Center, Hokkaido University, Sapporo, Japan

Correspondence to

Dr Kiyohide Fushimi;

kfushimi.hci@tmd.ac.jp

\section{ABSTRACT}

Objectives To verify the associations between participation in an in-hospital cardiac rehabilitation (CR) programme and clinical outcomes among patients with acute myocardial infarction (AMI) after percutaneous coronary intervention (PCI).

Design A retrospective cohort study using the Japanese administrative claims database.

Setting Japanese acute-care hospitals.

Participants Patients aged $\geq 18$ years who underwent $\mathrm{PCl}$ due to AMI and survived to discharge.

Primary and secondary outcome measure The primary outcomes were revascularisation, all-cause readmission and cardiac readmission (median follow-up period: 324 days, 236 days and 263 days, respectively). The secondary outcomes were all-cause mortality and cardiac mortality (median follow-up period: both were 460 days).

Result The data of 13697 patients were extracted from the database, and $65.4 \%$ of them participated in an in-hospital CR. The risks of revascularisation, allcause readmission and cardiac readmission among $C R$ participants were compared with those of non-participants using two statistical techniques: matched-pair analysis based on propensity score and a 30-day landmark analysis. The results of those analysis were consistent and showed that the CR participants had lower risk of revascularisation (adjusted HR: 0.74; 95\% Cl: 0.65 to 0.84 ), all-cause readmission (HR: $0.81 ; 95 \% \mathrm{Cl}: 0.74$ to 0.88 ) and cardiac readmission (HR: $0.77 ; 95 \% \mathrm{Cl}: 0.70$ to 0.85). However, all-cause mortality and cardiac mortality were not associated with participation in the CR. Conclusions It was suggested that in-hospital CR participation may reduce the risk of revascularisation, all-cause readmission and cardiac readmission among patients with $\mathrm{AMI}$ after $\mathrm{PCl}$. In-hospital CR may expand the potential benefits of $\mathrm{CR}$ in addition to outpatient $\mathrm{CR}$.

\section{INTRODUCTION}

Acute myocardial infarction (AMI) and other coronary artery diseases (CADs) have been the leading cause of death worldwide for more than 15 years and are one of the most socially burdensome diseases. ${ }^{1}$ Although the
Strengths and limitations of this study

- We investigated the association of clinical prognosis and early in-hospital CR programme, which has not been investigated widely, and found favourable association.

- We performed multiple analyses and obtained consistent results, indicating robustness of the results.

- The database used in this study includes a large number of inpatients in acute-care hospitals in Japan.

- We could not evaluate the important prognostic factors, including ejection fraction, exercise capacity, vital sign, functional status, frailty or social risk factors, because they were not included in the data we used.

- Due to the facility-based nature of the data, it was not possible to identify events that occurred outside of the hospital.

mortality of patients with AMI has declined in the past decade, ${ }^{2}$ approximately $20 \%$ of AMI survivors have experienced major adverse cardiac events within 1 year after hospital discharge. ${ }^{3}$ Therefore, the secondary preventive care of these patients is recognised to be important.

Cardiac rehabilitation (CR) is one of the secondary preventive care measures for patients with AMI, which is a multidisciplinary programme consisting of exercise therapy and patient education on secondary prevention and lifestyle modification. Many randomised controlled trials have been conducted, and meta-analysis of these results showed that CR reduced the mortality, readmissions and improved the management of risk factors. ${ }^{4-6}$ Based on this evidence, CR implementation is strongly recommended by relevant clinical guidelines. ${ }^{7-11}$ 
Since a major component of CR is the outpatient programme implemented after hospital discharge, the established benefits of CR are mainly dependent on the implementation of the outpatient programme. In the outpatient programme, participants start the programme after discharge and implement it regularly for weeks or months. In some cases, patients participate in the CR programme during hospitalisation. This in-hospital programme consists of programmes for prevention of deconditioning and recovery of daily activity in the acute phase, as well as supervised exercise therapy and patient education in the early recovery phase. ${ }^{9}$ Given that AMI survivors have a higher incidence rate of readmission or major adverse cardiac events early after discharge,${ }^{12-15}$ they need secondary preventive care immediately after onset. In addition, since an early enrolment in a CR programme is associated with a positive effect on exercise capacity, ${ }^{16}$ participation in the in-hospital CR programme may have beneficial effects on clinical outcomes. Some studies in Germany have reported that implementation of in-hospital CR improved risk factor modification and reduced all-cause mortality. ${ }^{17}{ }^{18}$ However, the associations of in-hospital CR and clinical prognosis such as revascularisations or readmissions are not fully revealed. In Japan, an in-hospital CR programme is predominantly conducted for patients with AMI based on the programme presented in the Japanese CR guideline. ${ }^{9}$

Here, we aimed to verify the associations between participation in an in-hospital CR programme and clinical outcomes among patients with AMI after percutaneous coronary intervention (PCI) on a nationwide scale using Japanese large administrative claims database. We hypothesised that in-hospital CR participation is related to the risk reduction of revascularisation and readmission after discharge.

\section{METHODS}

\section{Study design and data sources}

We conducted a retrospective cohort study using Japanese administrative data. The data were extracted from the Diagnosis Procedure Combination (DPC) database, which is a Japanese national case-mix classification system database. ${ }^{19}$ It consists of administrative claims data and discharge summary collected from acute-care hospitals that operate under the DPC system (DPC hospitals). The data amounts to 8 million inpatient episodes every year and contain all their acute-care inpatients' information. In this database, it is possible to link inpatient and outpatient records of each patient within each facility. All study procedures were performed in accordance with the Strengthening the Reporting of Observational Studies in Epidemiology statement. ${ }^{20}$ Patient consent was waived according to the Ethical Guidelines for Medical and Health Research Involving Human Subjects in Japan, ${ }^{21}$ as the data analysed were only anonymised secondary data. This study was approved by the Institutional Ethics
Committees at Tokyo Medical and Dental University (M2000-788).

\section{Study population}

We extracted data of patients aged $\geq 18$ years who underwent PCI for AMI and survived to discharge between 1 April 2012 and 31 March 2014 from the DPC database. In this study, we targeted patients who were admitted to the hospitals, authorised to provide CR and perform outpatient treatment of patients after discharge. The International Classification of Diseases, 10th Revision (ICD-10) codes, I21 and I22, were used to identify patients with AMI. If there were two or more records of admission, we defined the earliest record as index admission. The study included only patient who received outpatient care after discharge in the hospital; and excluded patients who stayed in the hospital for $>60$ days and who started in-hospital CR at 30 days or more after admission because they were considered highly likely to be clinical outliers. In addition, patients who participated in other rehabilitation programmes and who participated only in the outpatient CR programme were excluded to focus on the in-hospital CR.

Each patient was followed using all inpatient and outpatient records, which were created during the period from discharge until 31 March 2016. Considering the facilitybased data characteristics, only patients who had regular visits to the hospital were followed, and patients who completed their regular visits were treated as a censored case on the date of their last visit.

\section{Cardiac rehabilitation}

In Japan, CR is fully covered by the national health insurance. Because Japan has introduced the national public insurance system, all patients can receive CR within the health insurance. However, only authorised facilities meeting certain criteria can provide CR under the health insurance. The criteria include staffing requiring at least one full-time cardiologist or cardiac surgeon and dedicated full-time nurses or physical therapists with experience, equipment such as an ECG monitor, exercise equipment and defibrillator, and an emergency system that enables immediate surgery and angiography. Currently, there are approximately 1000 CR facilities in Japan; most of them are acute-care hospitals, and there are a few community-based facilities.

The CR programme usually starts within several days after admission and consists of a progressive exercise programme with an appropriate medical evaluation, aimed to help regain the ability of daily activities (Phase I programme). In about a week, it shifts continuously to the in-hospital Phase II programme, which is generally implemented on weekday during hospitalisation. In the programme, conducting risk assessment and exercise prescription based on the anaerobic threshold level or at $40 \%-60 \%$ of the heart rate reserve is recommended. The exercise intensity is recommended to be determined based on a submaximal cardiopulmonary 
exercise test performed 4-7 days after the onset. In addition to the exercise therapy, the programme also includes patient education and counselling. Along with the shortened hospitalisation, it is difficult to complete the CR programme during hospitalisation. Therefore, it is recommended that patients continuously participate in the outpatient CR programme after discharge. ${ }^{9} \mathrm{We}$ defined patients as in-hospital CR participants when they participated in the inpatient exercise-based CR program $\geq 1$ session within 30 days from admission. The participation status was ascertained using record of procedures in the DPC data. For in-hospital CR participants, we evaluated the number of days from admission to start of CR and the total number of sessions they have attended. We also evaluated the participation status of subsequent outpatient CR.

\section{Outcome}

The primary outcomes were time to revascularisation, all-cause readmission and cardiac readmission. Revascularisation was defined as a new PCI after the primary PCI was performed based on the clinical need. All-cause readmission was defined as a readmission after the index admission regardless of the main diagnosis. Cardiac readmission was defined as a readmission for cardiac disease, which was ascertained using codes I11, I13 and I20-I52 of the ICD-10 after the index admission. Scheduled readmissions within 4 weeks after discharge were not covered (1.0\% of CR participants and $3.4 \%$ of non-participants), because they could be a series of treatment from the primary PCI rather than a new onset of event. Moreover, readmissions for examination, including coronary angiography, and for education were not covered $(29.1 \%$ of CR participants and $23.1 \%$ of non-participants).

We also evaluated all-cause mortality and cardiac mortality as secondary outcomes. We defined all-cause death as death occurring in the hospital during readmission regardless of the main diagnosis, and cardiac death as death occurring in the hospital during readmission with cardiac disease as the main diagnosis.

\section{Other variables}

Clinical characteristics at admission were available from the inpatient records. Main diagnosis and comorbidities were evaluated with reference to the items corresponding to each disease name in DPC data. We evaluated the history of AMI, PCI and coronary artery bypass grafting $(\mathrm{CABG})$ within 1 year before the index admission using the inpatient records generated during the concerned period. Moreover, we evaluated the in-hospital and outpatient CR participation rates among patients with AMI who underwent PCI and the annual number of inpatients with AMI for each hospital as hospital factors.

\section{Statistical analysis}

The extracted patients were divided into two groups based on in-hospital CR participation. Differences in patients' characteristics between the two groups were compared using $\chi^{2}$ tests for binary variables, Wilcoxon's rank sum test for categorical variables and t-test for continuous variables.

To assess the association between participation in the in-hospital CR and study outcome variables, propensity score (PS) based matched-pair analysis was performed to produce a comparability between in-hospital CR participants and non-participants. We performed logistic regression analysis to create PS for the in-hospital CR participants. This model included the following variables: age, sex, body mass index (BMI), history of smoking, history of AMI, PCI, CABG (within 1 year before the admission), comorbidities (hypertension, dyslipidaemia, diabetes mellitus, heart failure, renal failure, cerebrovascular disease, peripheral artery disease and chronic obstructive pulmonary disease), Killip classification, length of hospital stay, medication (catecholamine, diuretic, aspirin, clopidogrel, $\beta$-blocker, statin and angiotensin converting enzyme (ACE) inhibitor/angiotensin receptor blocker (ARB)) and the annual number of inpatients with AMI. ${ }^{22}$ Then, PS for each patient for the probability of participation in the in-hospital CR was used to match participants and non-participants by using a oneto-one nearest neighbour matching with a calliper of 0.1 . For this matched pair, we estimated the incidence rates and performed a survival analysis using the Kaplan-Meier method and log-rank test for each outcome.

To confirm the robustness of the result with the abovementioned primary analysis, we performed an additional analysis. We applied Cox frailty model to calculate adjusted HRs by taking the effect of facilities into account as a random effect, and used all variables used for PS calculation in the models. We adopted the landmark analysis to increase comparability. ${ }^{23}$ We considered the 30-day mark as day 0 , and classified patients according to the CR participation status at 30 days after hospitalisation.

As sensitivity analysis, we performed a competing risk analysis for revascularisation, cardiac readmission and cardiac death, taking into account all-cause death as a competing risk. In addition, to assess the impact of severity on the association between in-hospital CR and primary outcomes, we estimated the HR by severity, which were as follows: severe group (Killip classification: $\geq 2$ ) and mild group (Killip classification: 1). HR was also calculated by using the Cox proportional hazard model with all variables used in the model of our primary analysis to clarify the result consistency. Lastly, the dose-response relationship between in-hospital CR and primary outcomes was evaluated. First, to determine the optimal cut-off number of sessions, a receiver operating characteristic curve was drawn for each three main outcomes. Next, the cut-off point was determined using the Youden's index. Based on the cut-off session number, the CR group was divided into the following two groups: a low-frequency group and a high-frequency group. Then, we performed Cox proportional hazard model with all variables mentioned above and estimated the adjusted HRs of both groups. 


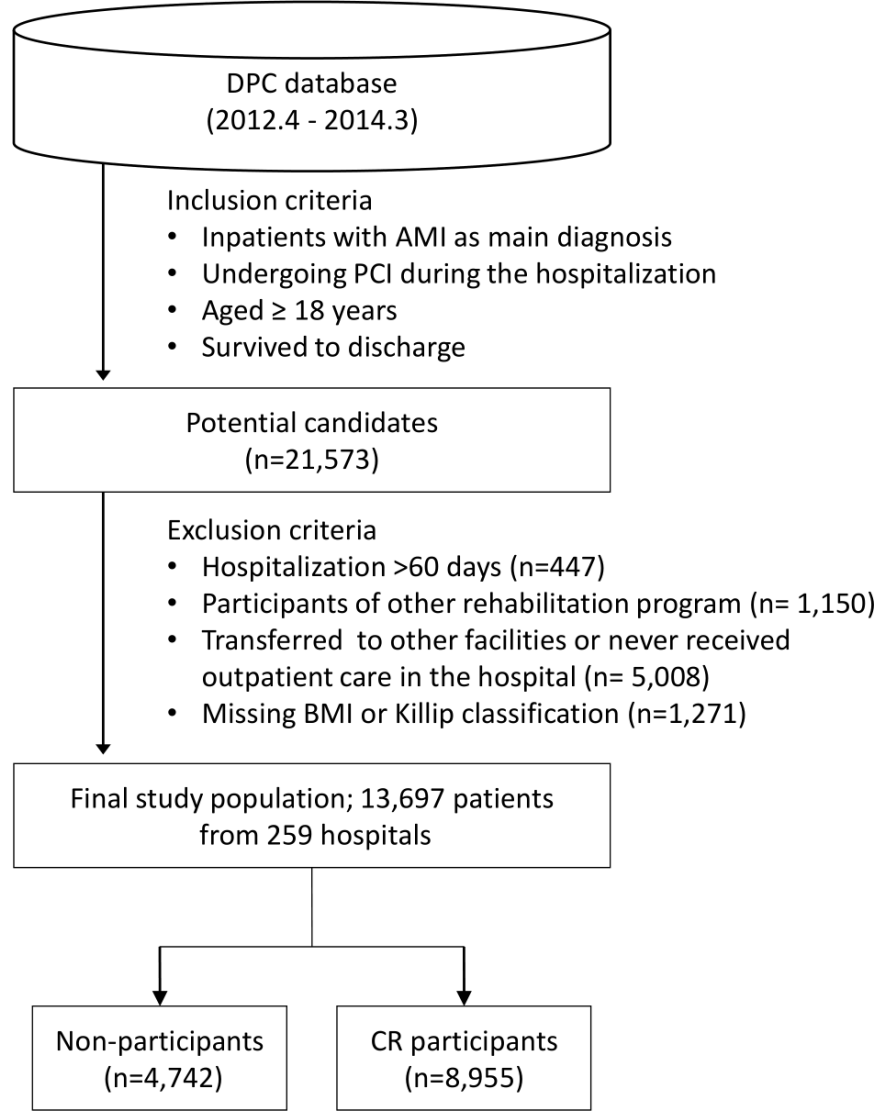

Figure 1 Flow diagram of the extraction process of the study population. AMI, acute myocardial infarction; BMI, body mass index; CR, cardiac rehabilitation; DPC, Diagnosis Procedure Combination; $\mathrm{PCl}$, percutaneous coronary intervention.

In this study, R V.3.5.0 was used to calculate PS and to perform the matched-pair analysis, and Stata V.14 software (Stata Corp LLC, College Station, Texas, USA) was used for all other analyses. A p value of 0.05 was considered significant in all analyses.

\section{Patient and public involvement}

Patients and the public were not directly involved in this study. The subjects of this study were identified from the DPC database, and the anonymised patient records in the database were used for the study.

\section{RESULTS}

\section{Patient characteristics and in-hospital CR participation}

A total of 21573 patients were extracted as potential candidates who underwent PCI due to AMI between 1 April 2012 and 31 March 2014. Applying the exclusion criteria, 13697 patients were eventually included in this study (figure 1). In-hospital CR participants accounted for $65.4 \%$ of the total subjects; of these, $7.6 \%(n=681)$ were post-discharge outpatient CR participants. Table 1 lists the main characteristics of the overall cohort and matched pair at baseline (all characteristics are shown in online supplemental table S1). In-hospital CR participants stayed longer in hospitals and were more likely to be treated with intra-aortic balloon pumping (IABP), catecholamine and diuretic than non-participants. The participants received more prescriptions for aspirin, clopidogrel, $\beta$-blocker, statin and ACE inhibitor/ARB at discharge than nonparticipants. This suggests that the in-hospital CR participants were more likely to have severe cardiac conditions and receive evidence-based pharmacotherapy than nonparticipants. The length of hospital stay, about 2 weeks, was much longer than recommended by European guidelines, ${ }^{7}$ which may represent a unique situation in Japan. The implementation status of CR programme is shown in table 2. Although there were large variations in time, in-hospital CR participants have undergone an average of 34 min of each session throughout their hospitalisation.

\section{Association between in-hospital CR and outcomes}

Revascularisation was noted in 2143 patients (median follow-up period: 324 days). All-cause readmission was noted in 5151 patients (median follow-up period: 236 days); of these, 3730 were cardiac readmissions (median follow-up period: 263 days). The 1-year follow-up rates of these outcomes were $59.8 \%, 65.6 \%$ and $63.6 \%$, respectively.

After PS matching between in-hospital CR participants and non-participants, 2441 pairs were generated. Distribution of each variable is listed in table 1 , which was successfully balanced. Matched-pair analysis showed that the incidence rates of revascularisation, all-cause readmission and cardiac readmission were significantly lower in the in-hospital CR participants than in the non-participants (figure 2 and table 3). The relative risks were $0.80,0.85$ and 0.81 , respectively. Conversely, there was no difference in the incidence rates of all-cause mortality and cardiac mortality between the two groups (median follow-up period: both were 460 days). The power of matched-pair analysis was maintained above 0.9 . The landmark analysis was performed on 12768 patients, and results are shown in figure 3. In the analysis, adjusted HR of revascularisation, all-cause readmission and cardiac readmission was significantly lower in the in-hospital CR participants than in the non-participants, which was slightly higher but consistent with the results of matched-pair analysis. Similar to the matched-pair analysis, there was no difference in all-cause mortality and cardiac mortality between the groups.

\section{Sensitivity analysis}

As a sensitivity analysis, we performed a competing risk analysis for revascularisation, cardiac readmission and cardiac death. The results were similar to the main results (see online supplemental table S2). Moreover, we estimated the adjusted HR by severity: 10811 patients and 1957 patients were categorised in the mild and severe groups, respectively (figure 3). The risks of revascularisation and readmission after PCI were consistently lower in the in-hospital CR participants than in the nonparticipants in any severity group.

There was a tendency of a dose-response relationship between in-hospital CR and main outcomes. We calculated 
Table 1 Baseline characteristics of overall cohort and matched-pair group

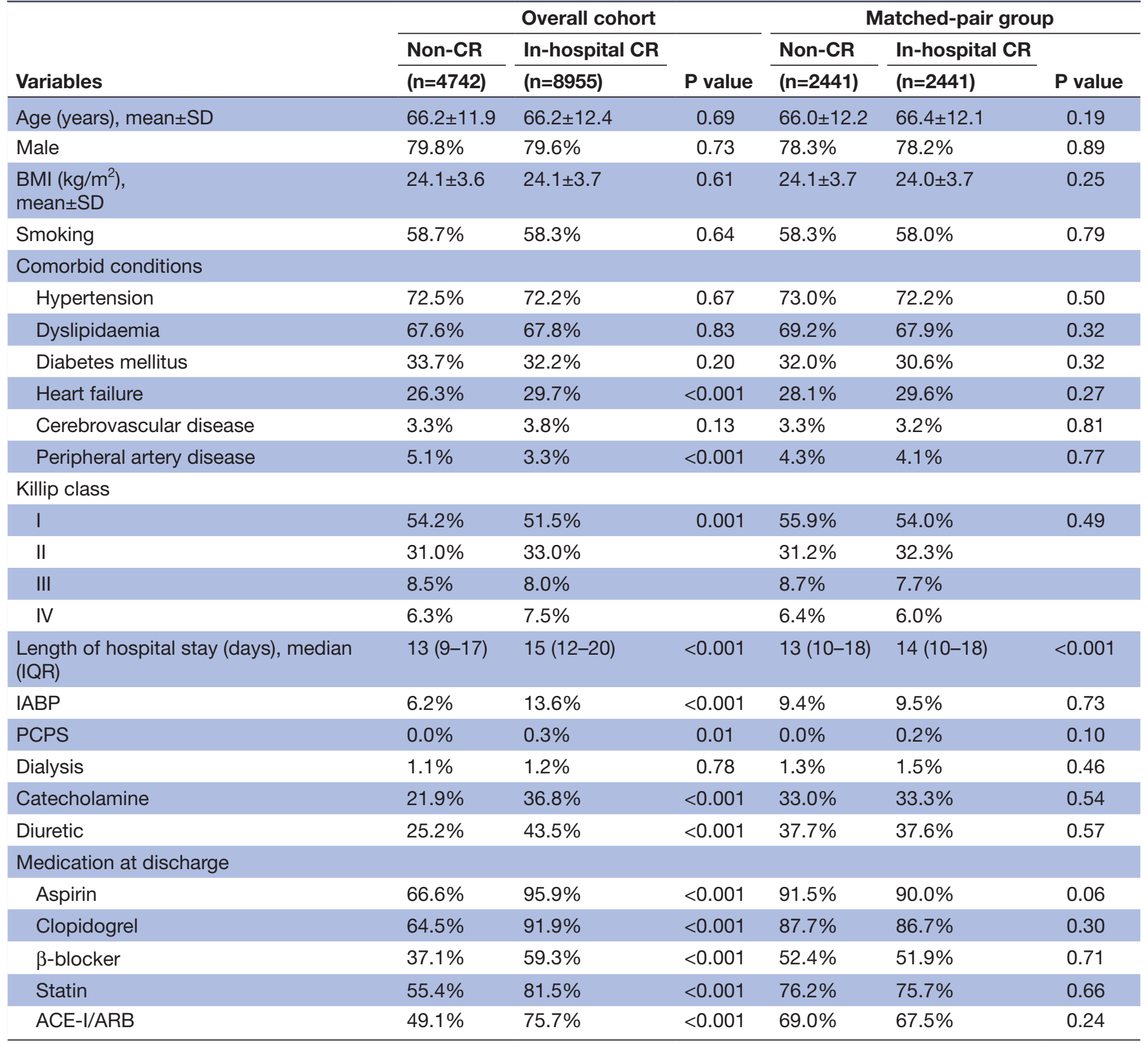

ACE-I, angiotensin converting enzyme inhibitor; ARB, angiotensin receptor blocker; BMI, body mass index; CR, cardiac rehabilitation; IABP, intra-aortic balloon pumping; PCPS, percutaneous cardiopulmonary support; SD, standard deviation.

Table 2 Implementation status of CR programmes

\begin{tabular}{lll} 
Implementation status & $\begin{array}{l}\text { In-hospital CR } \\
(\mathbf{n}=8955)\end{array}$ & $\begin{array}{l}\text { Outpatient CR } \\
(\mathbf{n}=681)\end{array}$ \\
\hline $\begin{array}{l}\text { Time to first participation } \\
\text { (days), median (IQR) }\end{array}$ & $3(2-6)$ & - \\
$\begin{array}{l}\text { The number of sessions, } \\
\text { median (IQR) }\end{array}$ & $7(5-10)$ & $6(8-12)$ \\
$\begin{array}{l}\text { Total time of attending } \\
\text { programme (min), median } \\
\text { (IQR) }\end{array}$ & $240(160-400)$ & $380(260-580)$ \\
\hline
\end{tabular}

$\mathrm{CR}$, cardiac rehabilitation; ;IQR, interquartile range. an optimal cut-off point of eight sessions, and divided the in-hospital CR participants into the low-frequency group $(\mathrm{n}=3951)$ and high-frequency group $(\mathrm{n}=5004)$ based on the number of sessions participated. Then, the risks for revascularisation and all-cause readmission tended to be lower in the high-frequency group than in the low-frequency group (table 4). On the other hand, the risk for cardiac readmission was not different between the two groups.

\section{DISCUSSIONS}

The study results showed that participation in the in-hospital CR programme was associated with $20 \%$ of risk reduction of revascularisation, $15 \%$ of all-cause 


\section{A}

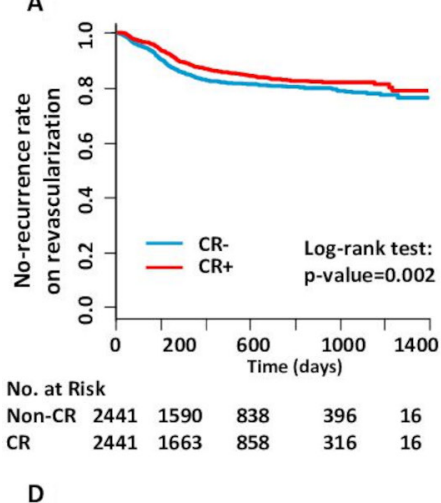

D

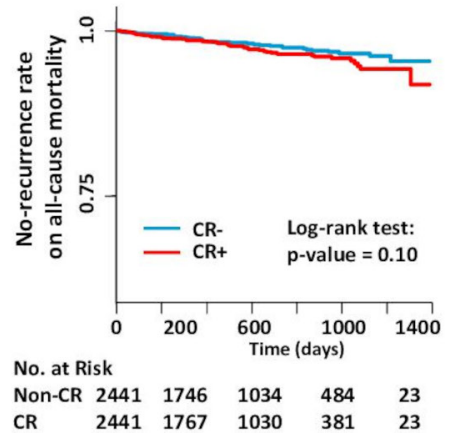

B

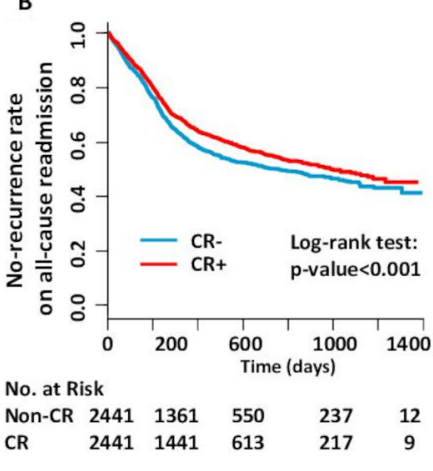

E

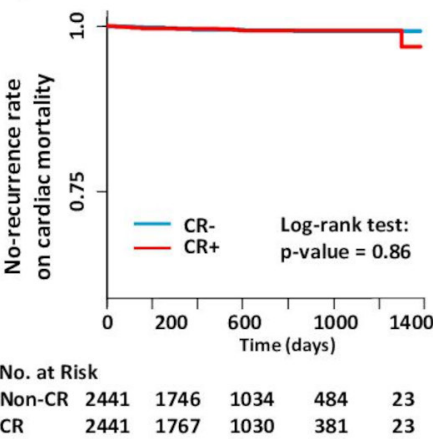

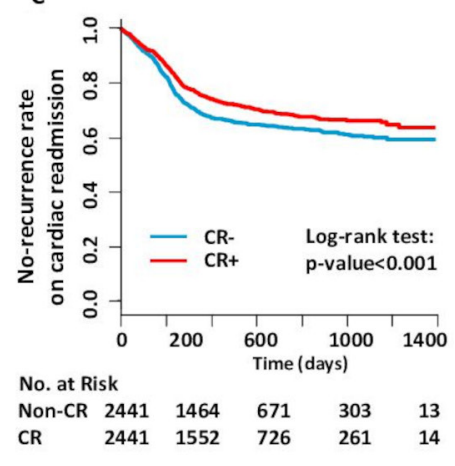

Figure 2 Kaplan-Meier curves and results of the log-rank test among the matched-pair groups. Each figure indicates the result of the outcomes such as (A) revascularisation, (B) all-cause readmission, (C) cardiac readmission, (D) all-cause mortality and (E) cardiac mortality. CR, cardiac rehabilitatiion.

readmission and $19 \%$ of cardiac readmission. Multiple analytic methods were used to confirm the robustness of these associations. These risk reductions were significant in terms of reduction of medical expenses and relief of patient burden. Given that the safety of the in-hospital CR programme has already been shown, ${ }^{24}$ the present study emphasised the potential benefit of the programme as secondary preventive care. However, all-cause mortality and cardiac mortality were not associated with the in-hospital CR participation.

Regarding the risk reduction of readmission, our results were consistent with those of conventional outpatient $\mathrm{CR},{ }^{5625}$ suggesting that the effect of CR on readmission may be consistent regardless of when the programme is delivered. In contrast, the risk reduction of revascularisation was limited to in-hospital CR, because it was not observed in outpatient CR. ${ }^{412}$ This indicated that there might be an additional benefit of early in-hospital CR. An in-hospital CR programme with exercise training enhanced the patient's exercise capacity after AMI, ${ }^{26}$ and improved the heart rate variability in patients with $\mathrm{CAD}$ after $\mathrm{CABG} .{ }^{27}$ In addition, participation in the inpatient exercise-based CR programme was related to increased physical activity at 1-year follow-up in elderly patients. ${ }^{28}$ Moreover, a study conducted in Germany reported the long-term salutary effects of in-hospital CR, such as significant reductions in systolic and diastolic blood pressure, better adherence to drug intake and fewer risk factors. ${ }^{17}$ The study also showed a strong tendency towards fewer recurrent cardiac events in the in-hospital CR group. ${ }^{17}$ Therefore, in-hospital CR programme with exercise training in early phases, even for a very short period, may improve physical and cardiac function, promote behavioural changes and control risk factors, thereby resulting in long-term favourable effects.

Table 3 Incidence rates of outcomes among matched pairs

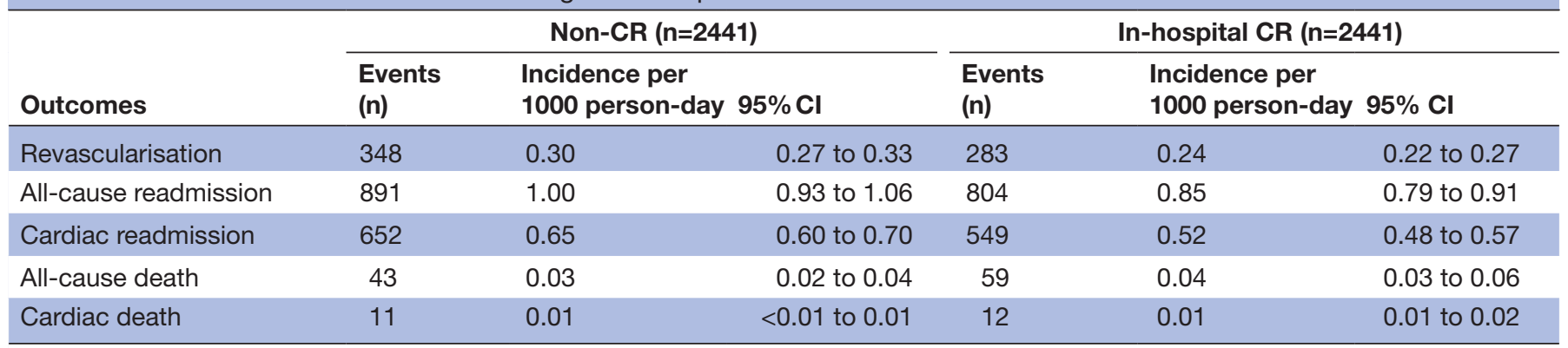

$\mathrm{CR}$, cardiac rehabilitation.; 


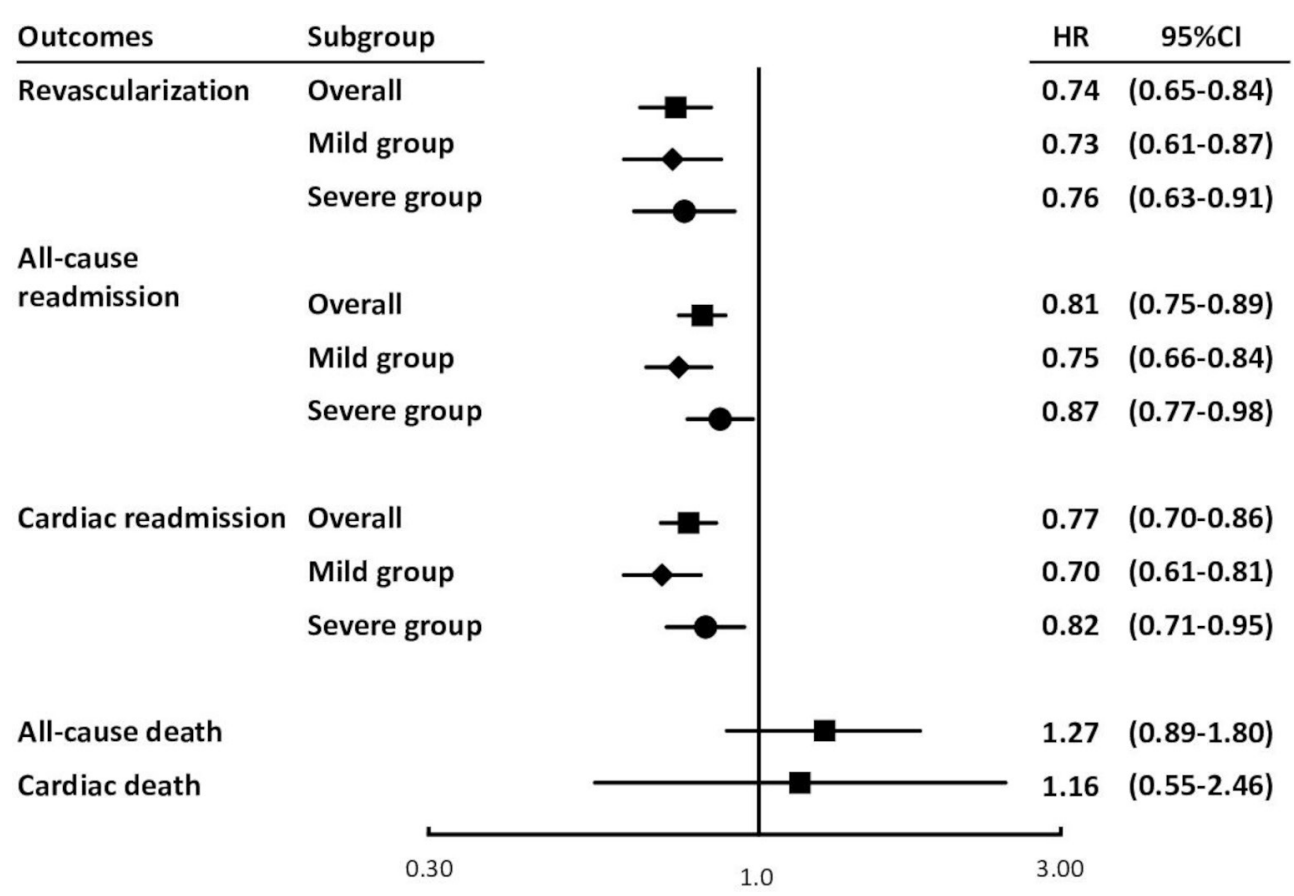

Figure 3 Adjusted $\mathrm{HR}$ and $95 \% \mathrm{Cl}$ for participants of the in-hospital cardiac rehabilitation after percutaneous coronary intervention due to acute myocardial infarction. The Cox proportional hazard models were performed excluding patients who experienced some events within 30 days after discharge or who were followed up $<30$ days (landmark analysis). For the primary outcomes, three HRs are shown: overall (indicated by $\square$ ) represents adjusted HR among all analysis objects; mild group (indicated by $\diamond$ ) represents adjusted HR for the mild group (Killip classification: 1 ) and severe group (indicated by $\mathbf{Q}$ ) represents adjusted HR for the severe group (Killip classifications: $\geq 2$ ).

To evaluate the dose-response relationship, a stratified analysis based on the participated session number was performed. The group with $\geq$ eight sessions tended to have greater risk reductions for revascularisation and all-cause readmissions than that with fewer sessions. This finding indicates that more intensive interventions might be associated with a greater impact on clinical prognosis. However, even the low-frequency CR group was associated with favourable clinical outcomes. In particular, the risk of cardiac readmission did not differ by frequency. It is unclear whether these results were due to the in-hospital CR programme itself or the combined impact of the in-hospital CR and subsequent improvements of lifestyle or adherence to the secondary preventive care, which was promoted by the in-hospital CR programme. Since we did not evaluate the exercise status, drug intake and diet after discharge, a further study is needed to clarify the underlying mechanisms.

In the matched-pair analysis, the Kaplan-Meier survival curves of revascularisation and readmissions were similar in the timing of divergence, suggesting that the majority of the events might be related to planned follow-up coronary angiography. Actually, the median number of days to the planned hospitalisation with coronary angiography was 232, which coincided with the time of divergence. Although these events were soft endpoints, which partly depend on the preference of both physicians and patients, the fact that there were at least some symptomatic or progressing stenosis requiring treatment should be acknowledged, and the prevention of these progressive conditions is clinically important as a goal of in-hospital CR.

Table 4 The relationship between participation frequency of in-hospital CR and risk reduction

\begin{tabular}{|c|c|c|c|c|}
\hline \multirow[b]{2}{*}{ Outcomes } & \multicolumn{2}{|c|}{$\begin{array}{l}\text { Low-frequency CR group } \\
\qquad(\mathrm{n}=3951)\end{array}$} & \multicolumn{2}{|c|}{$\begin{array}{l}\text { High-frequency CR group } \\
(\mathrm{n}=5004)\end{array}$} \\
\hline & HR & $95 \% \mathrm{Cl}$ & HR & $95 \% \mathrm{Cl}$ \\
\hline All-cause readmission & 0.84 & (0.77 to 0.90$)$ & 0.76 & (0.70 to 0.83 ) \\
\hline Cardiac readmission & 0.74 & (0.67 to 0.81$)$ & 0.76 & (0.69 to 0.84$)$ \\
\hline
\end{tabular}

The low-frequency group comprised of patients who participated in the CR programme for <eight sessions. The high-frequency group included patients who participated in the CR programme for zeight sessions.

$\mathrm{CR}$, cardiac rehabilitation. 
As secondary outcome, all-cause mortality and cardiac mortality were evaluated. The associations between participation in the in-hospital CR programmes and those outcomes were not observed in this study, which was not comparable with previous studies. ${ }^{13252930}$ One possible reason for this different finding may be the inadequate implementation. It is known that there is a dose-response relationship between the number of sessions and magnitude of the effect of CR on mortality. ${ }^{29}{ }^{31}$ Due to the small number of sessions in this study, reduction in mortality rates might not be observed in the participants. Rather, all-cause mortality tended to be higher in the CR group. Martin et al reported that the mortality in patients who did not complete 12-week CR programme and attended an average of 6.7 sessions tended to be slightly higher than that in non-CR attendees (HR: 1.08). ${ }^{13}$ The number of sessions and the tendency of the result were similar to those reported in our study. Since it is still unclear whether the small session number is related to increase in mortality, more precise investigation is needed.

In the process of patient selection, we excluded in-hospital death to investigate post-discharge event occurrence, which might cause immortality bias. We also excluded patients who were hospitalised for more than 60 days, initiated in-hospital CR at 30 days or more after admission, or never received outpatient care at the hospital. The comparison of patient characteristics between the overall cohort and the excluded patients is given in online supplemental table S3. The excluded patients were older, more likely to be men, with Killip classification $>2$, had longer hospitalisation, had renal disease and less likely to be prescribed medication at discharge. Whereas, the study population was more likely to have smoking history, comorbidities of hypertension and dyslipidaemia and use of catecholamine, diuretic and IABP. Which population had poorer prognosis was not clear, but if patients with more severe condition were selectively excluded, the results may be biassed towards better outcomes.

These findings suggested that early in-hospital CR is useful to reduce the risk of revascularisations and readmissions by initiating secondary preventive care early. However, the association with mortality was not observed. Therefore, in-hospital CR should not be considered an alternative model, but rather an additional model to the standard outpatient CR, which is well experienced and effective. Our results suggest that the early in-hospital CR model may expand the benefits of CR in combination with outpatient CR.

\section{Strengths and limitations}

A nationwide large-scale investigation is one of the strengths of this study. The DPC database includes a large number of inpatients in acute-care hospitals in Japan; thus, this study represents Japanese clinical settings of AMI well. Additionally, we reported the association of clinical prognosis and early in-hospital CR programme that has not been investigated widely. Japan is one of the few clinical sites where it is common to start
CR early in the hospitalisation process. The fact that the relationship between early CR and clinical outcomes was reported using Japanese large-scale data set is an important strength of this study. Moreover, our use of multiple analytical methods, PS matching and landmark analysis yielded consistent results, indicating their robustness.

There are several limitations to our study. First, the DPC data did not include detailed clinical data, such as ejection fraction, exercise capacity, vital sign, functional status, frailty or social risk factors, which are important prognostic factors. Although we evaluated the procedures, medications and all other available variables to adjust patient deviation, we could not adjust for imbalance in unmeasured confounders. Next, due to the facility-based nature of the DPC data, it was not possible to identify events that occurred outside of the hospital. Therefore, the incidence rate of each outcome may have been underestimated. In addition, if the occurrence of events outside the hospital is biased toward either group, the estimation of results may also be biased. To control for this bias, we limited the cohort to regularly visiting patients; however, the impact may not have been excluded completely. Furthermore, this process might have limited the cohort to patients with relatively good adherence and good prognosis. Also, we could not evaluate the outpatient CR participation status outside of the hospital. Although both CR group and non-CR group could include outpatient CR participants at other facilities, if CR group included such patients more, the result may not reflect the association with in-hospital CR itself, but in-hospital CR combined with outpatient CR. However, since the participation rate of outpatient CR programme was not high in Japan, ${ }^{32} 33$ we believe it is unlikely to have a significant impact on the overall results. Lastly, although our findings suggested the effect of early CR programme implemented in the in-hospital settings, it could not be extrapolated for outpatient setting. In addition, the results may only be applicable if the environment, including insurance coverage and inpatient care, is similar to that in Japan.

\section{CONCLUSIONS}

In this study, we revealed the association between early in-hospital CR and clinical outcomes among patients with AMI after PCI. The primary analysis with several sensitivity analyses yielded consistent results, showing that in-hospital CR participation was related to significant risk reduction of revascularisation, all-cause readmission and cardiac readmission among patients with AMI. Given that prevention of these events leads to reduction of patient burden and medical expenses, early in-hospital CR model may be useful model to expand the potential benefits of $\mathrm{CR}$ in addition to outpatient CR. Hence, similar to preventive drug therapy, in-hospital CR should be integrated in the general therapeutic management of AMI during hospitalisation. 
Contributors NK designed the study and wrote the research plan. KF extracted the data used for the study from the database, and NK made the dataset for the analysis. HI and NK undertook statistical analyses with feedback from KF. NK interpreted the results and wrote the first draft of the manuscript with critical comments and revision from $\mathrm{HI}$ and $\mathrm{KF}$.

Funding This work was supported by Grants-in-Aid for Scientific Research of Japan Society for the Promotion of Science grant number 16K21697 and Grantin-Aid for Research on Policy Planning and Evaluation from the Ministry of Health, Labor, and Welfare in Japan grant number 20AA2005.

Competing interests None declared.

Patient and public involvement Patients and/or the public were not involved in the design, or conduct, or reporting, or dissemination plans of this research.

Patient consent for publication Obtained.

Provenance and peer review Not commissioned; externally peer reviewed.

Data availability statement The data are available upon reasonable request. The data are deidentified participant data, and available from corresponding author KF (kfushimi.hci@tmd.ac.jp). The reuse of the data subset is permitted only for revalidation of the results.

Open access This is an open access article distributed in accordance with the Creative Commons Attribution Non Commercial (CC BY-NC 4.0) license, which permits others to distribute, remix, adapt, build upon this work non-commercially, and license their derivative works on different terms, provided the original work is properly cited, appropriate credit is given, any changes made indicated, and the use is non-commercial. See: http://creativecommons.org/licenses/by-nc/4.0/.

\section{ORCID iD}

Natsuko Kanazawa http://orcid.org/0000-0002-4405-4719

\section{REFERENCES}

1 World Health Organization. Global health estimates 2016: deaths by cause, age, sex, by country and by region, 2000-2016, 2018. Available: https://www.who.int/healthinfo/global_burden_disease/en/ [Accessed 13 Jul 2020]

2 Benjamin EJ, Virani SS, Callaway CW, et al. Heart disease and stroke Statistics-2018 update: a report from the American heart association. Circulation 2018;137:e67-492.

3 Jernberg T, Hasvold P, Henriksson M, et al. Cardiovascular risk in post-myocardial infarction patients: nationwide real world data demonstrate the importance of a long-term perspective. Eur Heart $J$ 2015;36:1163-70.

4 Taylor RS, Brown A, Ebrahim S, et al. Exercise-based rehabilitation for patients with coronary heart disease: systematic review and meta-analysis of randomized controlled trials. Am J Med 2004;116:682-92.

5 Heran BS, Chen JM, Ebrahim S, et al. Exercise-based cardiac rehabilitation for coronary heart disease. Cochrane Database Syst Rev 2011:CD001800.

6 Anderson L, Thompson DR, Oldridge N, et al. Exercise-based cardiac rehabilitation for coronary heart disease. Cochrane Database Syst Rev 2016:CD001800.

7 Ibanez B, James S, Agewall S, et al. 2017 ESC guidelines for the management of acute myocardial infarction in patients presenting with ST-segment elevation: the task force for the management of acute myocardial infarction in patients presenting with ST-segment elevation of the European Society of cardiology (ESC). Eur Heart J 2018;39:119-77.

8 O'Gara PT, Kushner FG, Ascheim DD, et al. 2013 ACCF/AHA guideline for the management of ST-elevation myocardial infarction: a report of the American College of cardiology Foundation/American heart association Task force on practice guidelines. Circulation 2013;127:e362-425.

9 JCS Joint Working Group. Guidelines for rehabilitation in patients with cardiovascular disease (JCS 2012). Circ J 2014;78:2022-93.

10 Kimura K, Kimura T, Ishihara M, et al. JCS 2018 guideline on diagnosis and treatment of acute coronary syndrome. Circ $J$ 2019;83:1085-196.
11 Ambrosetti M, Abreu A, Corrà U, et al. Secondary prevention through comprehensive cardiovascular rehabilitation: from knowledge to implementation. 2020 update. A position paper from the secondary prevention and rehabilitation section of the European association of preventive cardiology. Eur J Prev Cardiol 2020:204748732091337.

12 Goel K, Lennon RJ, Tilbury RT, et al. Impact of cardiac rehabilitation on mortality and cardiovascular events after percutaneous coronary intervention in the community. Circulation 2011;123:2344-52.

13 Martin B-J, Hauer T, Arena R, et al. Cardiac rehabilitation attendance and outcomes in coronary artery disease patients. Circulation 2012;126:677-87.

14 Kaneko H, Yajima J, Oikawa Y, et al. Impact of aging on the clinical outcomes of Japanese patients with coronary artery disease after percutaneous coronary intervention. Heart Vessels 2014;29:156-64.

15 Plüss CE, Billing E, Held C, et al. Long-term effects of an expanded cardiac rehabilitation programme after myocardial infarction or coronary artery bypass surgery: a five-year follow-up of a randomized controlled study. Clin Rehabil 2011;25:79-87.

16 Johnson DA, Sacrinty MT, Gomadam PS, et al. Effect of early enrollment on outcomes in cardiac rehabilitation. Am J Cardiol 2014;114:1908-11.

17 Baessler A, Hengstenberg C, Holmer S, et al. Long-term effects of in-hospital cardiac rehabilitation on the cardiac risk profile. A casecontrol study in pairs of siblings with myocardial infarction. Eur Heart J 2001;22:1111-8.

18 Jünger C, Rauch B, Schneider S, et al. Effect of early short-term cardiac rehabilitation after acute ST-elevation and non-ST-elevation myocardial infarction on 1-year mortality. Curr Med Res Opin 2010;26:803-11.

19 Matsuda S, Ishikawa BK, Kuwabara K, et al. Development and use of the Japanese case-mix system. Eurohealth 2008;14:27-30.

20 STROBE Statement. Institute of social and preventive medicine, 2009. Available: https://www.strobe-statement.org/index.php?id= strobe-home [Accessed 22 Jan 2020].

21 Japanese Ministry of Health, Labor and Welfare. Ethical guidelines for medical and health research involving human subjects, 2015. Available: https://www.mhlw.go.jp/file/06-Seisakujouhou-10600000-Daijinkanbou kouseikagakuka/0000080278.pdf [Accessed 22 Jan 2020].

22 Doll JA, Hellkamp A, Thomas L, et al. Effectiveness of cardiac rehabilitation among older patients after acute myocardial infarction. Am Heart J 2015;170:855-64.

23 Anderson JR, Cain KC, Gelber RD. Analysis of survival by tumor response. J Clin Oncol 1983;1:710-9.

24 Saito M, Ueshima K, Saito M, et al. Safety of exercise-based cardiac rehabilitation and exercise testing for cardiac patients in Japan: a nationwide survey. Circ J 2014;78:1646-53.

25 Dunlay SM, Pack QR, Thomas RJ, et al. Participation in cardiac rehabilitation, readmissions, and death after acute myocardial infarction. Am J Med 2014;127:538-46.

26 Tsai Y-J, Li M-H, Chen C-H, et al. Improved oxygen uptake efficiency slope in acute myocardial infarction patients after early phase I cardiac rehabilitation. Int J Rehabil Res 2017;40:215-9.

27 Mendes RG, Simões RP, De Souza Melo Costa F, et al. Shortterm supervised inpatient physiotherapy exercise protocol improves cardiac autonomic function after coronary artery bypass graft surgery--a randomised controlled trial. Disabil Rehabil 2010;32:1320-7.

28 Macchi C, Polcaro P, Cecchi F, et al. One-year adherence to exercise in elderly patients receiving postacute inpatient rehabilitation after cardiac surgery. Am J Phys Med Rehabil 2009;88:727-34.

29 Suaya JA, Stason WB, Ades PA, et al. Cardiac rehabilitation and survival in older coronary patients. J Am Coll Cardiol 2009;54:25-33.

30 Beauchamp A, Worcester M, Ng A, et al. Attendance at cardiac rehabilitation is associated with lower all-cause mortality after 14 years of follow-up. Heart 2013;99:620-5.

31 Hammill BG, Curtis LH, Schulman KA, et al. Relationship between cardiac rehabilitation and long-term risks of death and myocardial infarction among elderly Medicare beneficiaries. Circulation 2010;121:63-70.

32 Goto Y. Current state of cardiac rehabilitation in Japan. Prog Cardiovasc Dis 2014;56:557-62.

33 Kanazawa N, Ueshima K, Tominari S, et al. Underuse of cardiac rehabilitation in workers with coronary artery disease - claims database survey in Japan. Circ J 2017;81:1424-31. 